\title{
A Study on the Effect of Plasma Treatment for Waste Wood Biocomposites
}

\author{
MiMi Kim, Heung Soo Kim, and Joong Yeon Lim \\ Department of Mechanical, Robotics and Energy Engineering, Dongguk University-Seoul, 26 Pil-dong 3-ga, Jung-gu, \\ Seoul 100-715, Republic of Korea
}

Correspondence should be addressed to Joong Yeon Lim; jylim@dongguk.edu

Received 12 June 2013; Accepted 3 July 2013

Academic Editor: Il-Kwon Oh

Copyright (C) 2013 MiMi Kim et al. This is an open access article distributed under the Creative Commons Attribution License, which permits unrestricted use, distribution, and reproduction in any medium, provided the original work is properly cited.

The surface modification of wood powder by atmospheric pressure plasma treatment was investigated. The composites were manufactured using wood powder and polypropylene (wood powder: polypropylene $=55 \mathrm{wt} \%: 45 \mathrm{wt} \%$ ). Atmospheric pressure plasma treatment was applied under the condition of $3 \mathrm{KV}, 17 \pm 1 \mathrm{KHz}, 2 \mathrm{~g} / \mathrm{min}$. Helium was used as the carrier gas and hexamethyldisiloxane (HMDSO) as the monomer to modify the surface property of the waste wood biocomposites by plasma polymerization. The tensile strengths of untreated waste wood powder (W3) and single species wood powder (S3) were about 18.5 MPa and $21.5 \mathrm{MPa}$ while those of plasma treated waste wood powder (PW3) and plasma treated single species wood powder (PS3) were about $21.2 \mathrm{MPa}$ and $23.4 \mathrm{MPa}$, respectively. Tensile strengths of W3 and S3 were improved by $14.6 \%$ and $8.8 \%$, respectively. From the analyses of mechanical properties and morphology, we conclude that the interfacial bonding of polypropylene and wood powder can be improved by atmospheric pressure plasma treatment.

\section{Introduction}

Governmental regulations and growing environmental awareness worldwide have triggered a paradigm shift towards designing materials compatible with the environment [1]. Biocomposites are most actively investigated as environment friendly materials [2]. Biofibers or natural fibers have been generally used as reinforcements for biocomposites. The matrices of biocomposites are biodegradable or nonbiodegradable polymers that can be extracted from natural resources instead of existing oil resources [3].

Wood plastics composites (WPCs) are made of wood powder. Composites are made by combining two materials to obtain better performance than either of the original ones while the original phases of these materials are retained [4]. Strong interfacial adhesion between fibers and matrices, however, is very important in making composites with high mechanical properties. For production of WPCs, the two materials that are to be combined must be treated by coupling agents because of the incompatibility between the hydrophilic wood powder and the hydrophobic polymer. However, the high prices of coupling agents have reduced the productivity of WPCs. Moreover, coupling agents are chemical materials that are not environment friendly. Therefore, new technologies have been developed to produce environment friendly materials from biomass resources. The interfacial adhesion in biocomposites can be enhanced by two types of methods: one type modifies the base polymers and another type treats the biodegradable fillers to increase their hydrophobic properties. The latter type includes methods such as corona treatment, plasma treatment, heating, and so forth [5]. Plasma treatment changes the chemical structure and surface properties of hydrophilic natural fibers to give them hydrophobic properties, which would enhance their interfacial adhesion with the base polymer $[6,7]$.

For mass production of biocomposites, atmospheric pressure plasma was used in this study instead of vacuum plasma. Biocomposites production in an atmospheric pressure plasma is a dry process that has many advantages such as low contamination, energy savings, and enhanced interfacial adhesion $[8,9]$. It also removes foreign matters by making chemical reactions only on surfaces of polymer while preserving the basic physical properties of the polymer [10]. Plasma processes, which have been usually carried out 
in vacuum, are now carried out at atmospheric pressure, and these atmospheric pressure plasma processes have become very popular because of their reduced production cost and shortened process time. Recently, the application of atmospheric pressure plasma processes has been extended. Plasma processes in vacuum, on the other hand, take a long time because of the less numbers of electrons and ions in vacuum plasma than in atmospheric pressure plasma, and they also have difficulty in providing stable and uniform modification.

In this study, we tried to make biocomposites with increased interfacial adhesion between the wood power and the polypropylene (PP) of hydrophobic thermoplastic polyolefin polymers by modifying the surfaces of the hydrophilic wood powder particles. To achieve this objective, a lab scale chamber was made. A screw was inserted in the chamber to move the wood powder. Electrodes were installed outside the chamber so that the wood powder could move and plasma processes could be carried out by rotating the screw. Continuity and good productivity were achieved.

The surfaces of the wood powder particles are modified by plasma processes as follows. First, electrons and gas molecules collide in the plasma. When an electron collides with a molecule XY, an electron escapes from the molecule $\mathrm{XY}$ and the molecule becomes ionized. Sometimes, the bond of the molecule $\mathrm{XY}$ is broken, and the molecule divides into $\mathrm{X}$ and $\mathrm{Y}$. The divided molecules $\mathrm{X}$ and $\mathrm{Y}$ are then likely to interact with the many unbounded electrons. These molecules are called radicals or chemically-active species. Through the chemical reactions of the ionized electrons in collisions in the plasma, wood powder becomes coated and the dispersion components on the surfaces are increased. Then, the surfaces of the wood power particles are modified to have hydrophobicity. To investigate such effects of surface modification, experiments were carried out for waste wood powder and single species wood powder. If the interfacial adhesion of waste wood powder with polypropylene is enhanced by surface modification, the strength of the waste wood biocomposites would increase and the waste wood powder could be used as a recycled raw material of biocomposites.

\section{Experimental Procedure}

2.1. Materials. Two types of wood powder were used: one was a single species powder of spruce and the other was a waste wood powder of crushed, construction waste wood chips. Wood powder was extracted from a 60-80 mesh sieve. Before wood powder was treated by plasma, it was dried in vacuum at moisture content of less than $1 \%$. Hexamethyldisiloxane (HMDSO) was used in the plasma treatment to modify the surface properties of the wood powder to make it hydrophobic. We used H1501 Polypropylene (LG Chem.) of less than $3 \mathrm{~mm}$ in diameter, $0.9 \mathrm{~g} / \mathrm{cm}^{3}$ in density, and $17 \mathrm{~g} / 10 \mathrm{~min}$ in melting index.

2.2. Contact Angle. A series of experiments were conducted to select the monomer that would be used to modify the hydrophilic wood powder surface into a hydrophobic surface.
A film type of polyethylene terephthalate (PET) was produced using a press. The plasma treatment was conducted using a vacuum plasma. The monomer used in the experiment was evaporated and then used to modify the PET film surface. Ten microliters of water and glycerol droplets was dropped on the processed film using the sessile drop method, and then the contact angle was measured. All contact angle measurements were repeated three times to obtain the mean value and the deviation.

2.3. Atmospheric Pressure Plasma Treatment. In the atmospheric pressure plasma treatment for the modification of the wood powder surface, helium was used to make the plasma generation atmosphere and as the carrier gas. Wood powder, injected through a hopper, was moved by a screw inserted at the chamber. The plasma generated between the two types of electrodes surrounding the chamber treated the wood powder being moved by the screw. At this time, the plasma treatment condition was $3 \mathrm{kV}$ at $17 \pm 1 \mathrm{kHz}$. Figure 1 shows schematic of the atmospheric pressure plasma treatment.

2.4. Composite Processing. For the mechanical mixing of wood powder and polypropylene, pellets were produced at $210^{\circ} \mathrm{C}$ at $180 \mathrm{rpm}$ using a twin screw extruder. The wood powder content in the biocomposites was fixed at $55 \mathrm{wt} \%$. The dispersion of the wood powder in PP was increased by carrying out the extrusion process three times. The pellets produced were dried for 24 hours at $60^{\circ} \mathrm{C}$, and then, the specimens whose mechanical properties were to be evaluated were injected by the injection molding machine.

2.5. Mechanical Properties. The tensile test was conducted at $5 \mathrm{~mm} / \mathrm{min}$ at room temperature using a $100 \mathrm{kN}$ load according to ASTM D638, while the flexural test was conducted at $3.4 \mathrm{~mm} / \mathrm{min}$ at room temperature using a $100 \mathrm{kN}$ load according to ASTM D790. Five specimens were tested for each experiment. The average value of the measurements was calculated and used for analysis.

\section{Results and Discussion}

3.1. Surface Free Energy. The intermolecular forces acting on the surface of the polymer can be divided into three categories: primary (chemical) force, quasi-chemical (hydrogen bond) force, and secondary (Van der Waals) force. The secondary force is again subdivided into Keesom force, Debye force, and London dispersion force, which is much larger than the other two Van der Waals forces. Surface tension is surface free energy per unit area, thermodynamically. Interfacial tension is created by cohesion and adhesion, which are the results of intermolecular forces at the interface of two different molecules. Since the free energy of a solid surface cannot be measured directly, unlike that of a liquid, it is calculated indirectly using a liquid whose surface tension is known.

Surface energy can be expressed in Young-Dupre' equation as follows. 


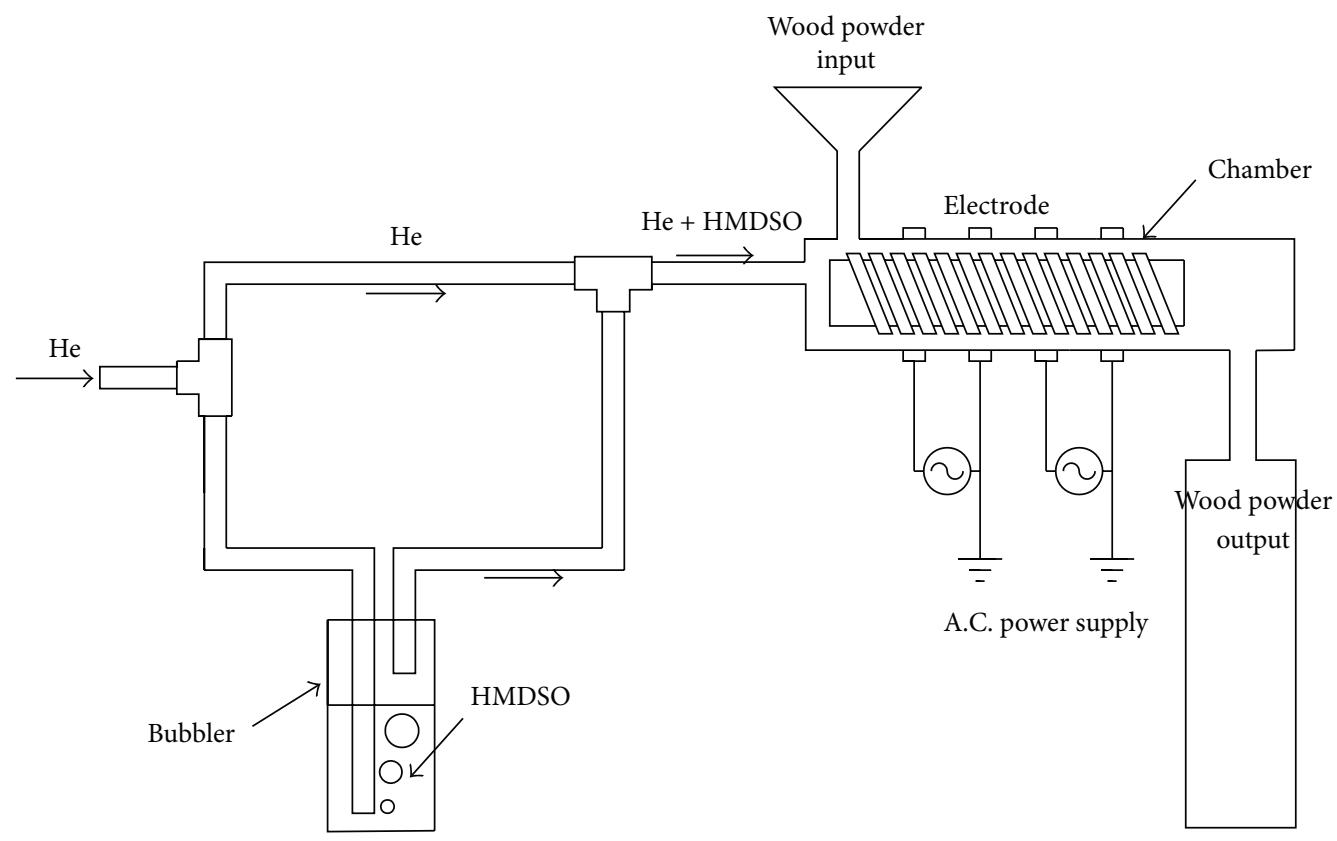

FIGURE 1: Schematic of atmospheric pressure plasma treatment.

Young equation:

$$
\gamma_{\mathrm{SV}}=\gamma_{\mathrm{SL}}+\gamma_{\mathrm{LV}} \cos \theta
$$

Dupre equation:

$$
W_{A}=\gamma_{\mathrm{LV}}+\gamma_{\mathrm{SV}}-\gamma_{\mathrm{SL}}
$$

Young-Dupre equation:

$$
W_{A}=\gamma_{\mathrm{LV}}(1+\cos \theta) \text {. }
$$

In addition, it can be expressed as the sum of polar components and dispersion components. Consider the following:

$$
\gamma_{\mathrm{SL}}=\gamma_{\mathrm{SV}}+\gamma_{\mathrm{LV}}-2\left(\gamma_{S}^{d} \gamma_{\mathrm{LV}}^{d}\right)^{1 / 2}-2\left(\gamma_{S}^{p} \gamma_{\mathrm{LV}}^{P}\right)^{1 / 2}
$$

Contact angle is a measure that represents the wettability of a solid surface. It can be calculated by measuring the contact angle of a water droplet on the PET film surfacemodified by plasma treatment. In general, a low contact angle indicates high wettability (hydrophilic property), and a high contact angle represents low wettability (hydrophobic property). Figure 2 shows equilibrium contact angle of a solid/liquid surface.

Table 1 provides the measured contact angle and surface energy of the PET film. Experimental results showed that the plasma-treated film had a low contact angle and a high surface energy but that the polar components were much higher than the dispersion components. These results confirm the modification of the film surface into that having the hydrophilic property.

This experiment aims to modify the hydrophilic surface of wood powder into a hydrophobic surface. The suitable monomer is hexamethyl-disiloxane (HMDSO). The polar
TABLE 1: Contact angle and surface energy of PET film.

\begin{tabular}{lccccc}
\hline \multirow{2}{*}{ Monomer } & \multicolumn{3}{c}{ Contact angle $\left(^{\circ}\right)$} & \multicolumn{3}{c}{ Surface energy $($ dyne/cm) } \\
& Water & Glycerol & Total & Dispersion & Polar \\
\hline Oxygen/PET & 8 & 9 & 73.73 & 12.78 & 60.96 \\
Benzene/PET & 58.8 & 65 & 49.17 & 1.08 & 47.37 \\
$\mathrm{CH}_{4} / \mathrm{PET}$ & 55.7 & 47.6 & 59.56 & 2.14 & 57.42 \\
$\begin{array}{l}\text { Acrylic acid/PET } \\
\mathrm{HMDSO} / \mathrm{O}_{2}\end{array}$ & 35.7 & 47.7 & 71.94 & 1.72 & 70.22 \\
$\begin{array}{l}1: 9) / \mathrm{PET} \\
\mathrm{HMDSO} / \mathrm{O}_{2}\end{array}$ & 28 & 41 & 75.74 & 2.54 & 73.21 \\
$\begin{array}{l}(2: 8) / \mathrm{PET} \\
\mathrm{HMDSO} / \mathrm{O}_{2}\end{array}$ & 66.5 & 60 & 47.25 & 4.32 & 42.93 \\
$\begin{array}{l}(3: 7) / \mathrm{PET} \\
\mathrm{HMDSO} / \mathrm{O}_{2}\end{array}$ & 72 & 73 & 32.39 & 4.57 & 27.81 \\
$\begin{array}{l}(4: 6) / \mathrm{PET} \\
\mathrm{HMDSO} / \mathrm{O}_{2}\end{array}$ & 73.5 & 73.5 & 30.64 & 5.43 & 25.21 \\
$\begin{array}{l}(5: 5) / \mathrm{PET} \\
\mathrm{HMDSO} / \mathrm{PET}\end{array}$ & 105 & 76 & 74.77 & 71.68 & 3.09 \\
\hline
\end{tabular}

component among the surface energy of the film after HMDSO plasma treatment was very low, which was also verified by measurement of the contact angle. However, oxygen mixing ratio also plays an important role in plasma treatments using HMDSO. The values of the plasma-treated polar component and dispersion component obtained by mixing HMDSO and oxygen at a mixing ratio of $1: 9$ showed the opposite of those when treated using HMDSO alone. As a result, it was found that HMDSO was most effective when modifying a surface to have hydrophobic property, but the results depend on the amount of oxygen input. 


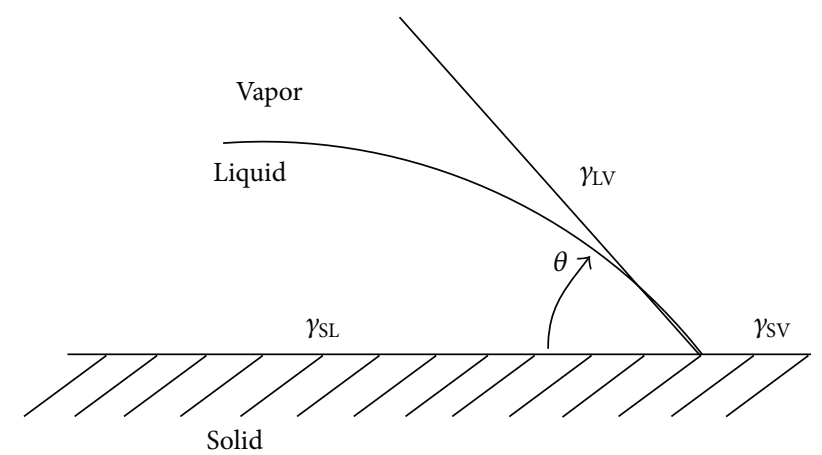

FIGURE 2: Equilibrium contact angle of solid/liquid surface.

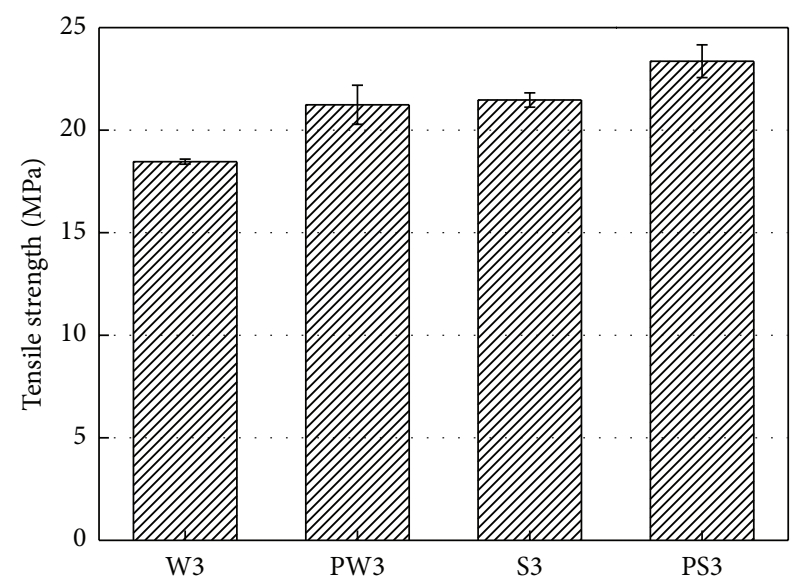

W3-waste wood powder PW3-waste wood powder by plasma treatment S3-single species wood powder PS3-single species wood powder by plasma treatment

FIgURE 3: Tensile strength of composites.

3.2. Tensile Strength. Figures 3 and 4 show the effect of plasma treatment on the tensile strength and tensile modulus of the composites according to the type of wood powder. The tensile strength of the composites formed from the single species wood powder was $21.5 \mathrm{MPa}$, which is $16.2 \%$ higher than that of the composites formed from the waste wood powder $(18.5 \mathrm{MPa})$. The reason why the tensile strength of the composites made of the waste wood powder was lower than that of the composites made of the single species wood powder was due to the foreign matters in the waste wood powder. These foreign matters come from the surface overlay, coating and adhesives of living wood waste and construction waste woods, which are the raw materials of waste wood powder. The foreign matters make recycling waste wood difficult. The strength of waste wood powder should be improved to broaden its application. The surface of waste wood powder can be modified to increase the strength of waste wood composites. The experimental results showed that the tensile strength of waste wood powder after surface modification by the atmospheric pressure plasma treatment was $21.2 \mathrm{MPa}$, which is an increase of $14.6 \%$ from that of the

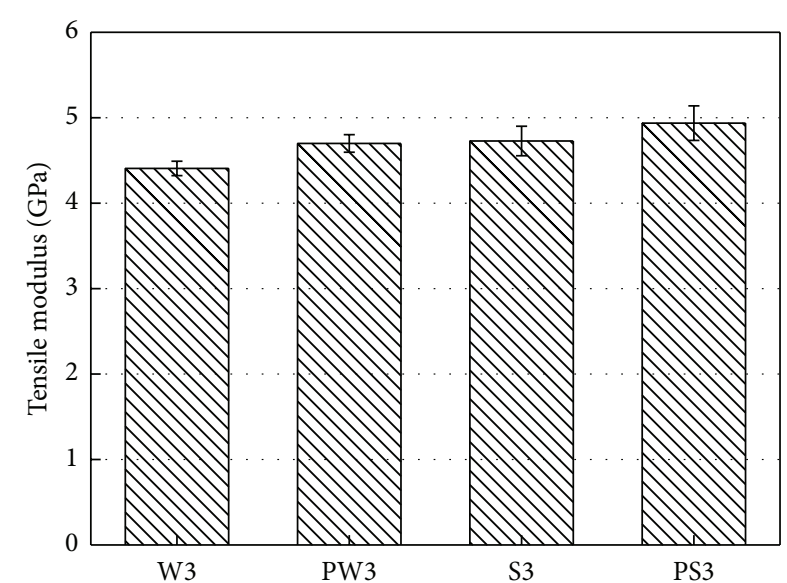

W3-waste wood powder PW3-waste wood powder by plasma treatment S3-single species wood powder PS3-single species wood powder by plasma treatment

FIgURE 4: Tensile modulus of composites.

TABLE 2: Flexural strength and flexural modulus of composites.

\begin{tabular}{lcccc}
\hline & W3 & PW3 & S3 & PS3 \\
\hline Flexural strength $(\mathrm{MPa})$ & 36.0 & 39.5 & 41.1 & 42.6 \\
Flexural modulus $(\mathrm{GPa})$ & 3.8 & 4.0 & 4.3 & 4.5 \\
\hline
\end{tabular}

nontreated waste wood powder. The plasma-treated single species wood powder also showed an increase in tensile strength. These results confirm that atmospheric pressure plasma treatment is effective in the surface modification of wood powder. The tensile modulus of the single species wood powder was higher than that of the waste wood powder. They were also increased by plasma treatment.

3.3. Flexural Strength. The flexural strength and flexural modulus of the composites according to the type of wood powder are summarized in Table 2 . The flexural strength of the composite made from the single species wood powder was $41.1 \mathrm{MPa}$, which is $14.2 \%$ higher than that of the composite made from the waste wood powder $(36.0 \mathrm{MPa})$. It was also observed that the flexural strength of the composite made from the waste wood powder with surface modification by atmospheric pressure plasma treatment was $39.5 \mathrm{MPa}$, which is an increase of $9.7 \%$ from that made from the nontreated waste wood powder. In addition, flexural moduli also increased in the single species wood powder.

3.4. SEM Images. Figures 5 and 6 present the cross-sections of the composites observed by SEM. In the case of the composite made of the nontreated wood powder, voids were observed at the interface between the wood powder and polymer. However, voids were not observed at the interface in the case of the composite made of the plasma-treated wood powder. These results showed that the hydrophilic surface of the wood powder was modified into a hydrophobic surface, which 


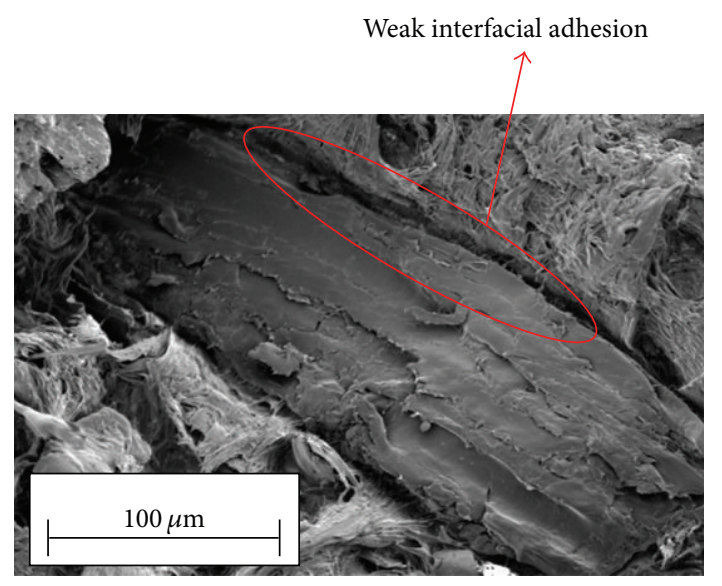

FIGURE 5: SEM micrograph of nontreated composites' cross-section.

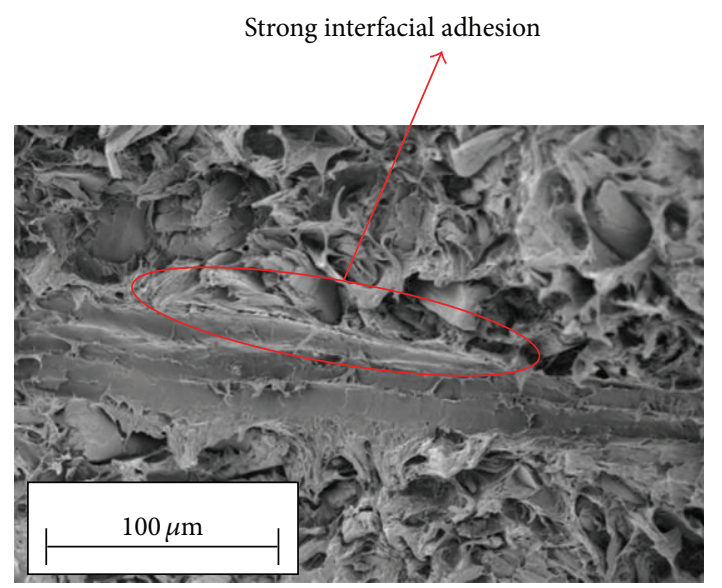

FIGURE 6: SEM micrograph of plasma-treated composites' crosssection.

helped to improve the interfacial adhesion with polymer, which is hydrophobic. The experimental results of tensile and flexural strengths also provided the same tendency.

\section{Conclusion}

The strengths of waste wood biocomposites were investigated for recycling waste wood. The surfaces of the wood powders were modified to enhance their interfacial adhesion with polypropylene by atmospheric pressure plasma treatment. Several important observations were made from the tensile and flexural strength tests as follows.

(1) Although the total energy of the surfaces was high by the atmospheric pressure plasma treatment using monomers, the surfaces were modified into hydrophobic or hydrophilic surfaces depending on polar or dispersed components.

(2) The most efficient monomer for the modification of the hydrophilic wood powders was HMDSO, which had the lowest polar component $(3.09$ dyne $/ \mathrm{cm})$ in the surface energy calculated by measurement of the contact angle.

(3) Tensile strengths of the composites made of the waste wood powder and single species wood powder were $18.5 \mathrm{MPa}$ and $21.5 \mathrm{MPa}$, respectively. The tensile strength of the single species wood powder composites was $16.2 \%$ higher than that of the waste wood powder composites. Foreign matters on the wood powder surface decreased the tensile strength of the waste wood powder composites. This is why plasma treatment is required for recycling waste wood.

(4) When the surface of the waste wood powder was modified by atmospheric pressure plasma treatment, the tensile strength increased by $14.6 \%$ from $18.5 \mathrm{MPa}$ to $21.2 \mathrm{MPa}$ and the flexural strength also increased by $9.7 \%$, from $36.0 \mathrm{MPa}$ to $39.5 \mathrm{MPa}$. In the case of the single species wood powder, the tensile strength increased by $8.8 \%$ and flexural strength increased by $3.6 \%$. These results confirm that surface modification by atmospheric pressure plasma treatment is effective for increasing the strength of both single species wood powder and waste wood powder.

(5) Through surfaces' modification by plasma treatment, the mechanical properties of the waste wood powder became similar to those of the single species wood powder without modification. Therefore, it is expected that waste wood powder can be recycled as raw biomaterials by various treatment processes.

\section{Acknowledgments}

This research was supported by the Ministry of Land, Infrastructure and Transport and the Korea Agency for Infrastructure Technology Advancement.

\section{References}

[1] A. K. Mohanty, M. A. Khan, and G. Hinrichsen, "Surface modification of jute and its influence on performance of biodegradable jute-fabric/Biopol composites," Composites Science and Technology, vol. 60, no. 7, pp. 1115-1124, 2000.

[2] S.-Y. Park, G.-S. Han, H.-S. Kim, H.-S. Yang, and H.-J. Kim, "Evaluation of the impact on manufacturing temperature and time in the production process of bio-composites," Mokchae Konghak, vol. 33, no. 1, pp. 29-37, 2005.

[3] T. Takayama, K. Komabayasi, M. Itou, and Y. Miyake, "Development of Bio-based plastics for injection molding," $S A E$ International Journal of Materials and Manufacturing, vol. 2, no. 1, pp. 12-17, 2009.

[4] C.-H. Kim, K.-J. Kim, and T.-J. Eom, "Properties of WPC prepared with various size and amount of wood particle," Palpu Chongi Gisul/Journal of Korea Technical Association of the Pulp and Paper Industry, vol. 40, no. 3, pp. 59-64, 2008.

[5] B.-H. Lee, H.-S. Kim, S.-W. Choi, and H.-J. Kim, "Improvement of interfacial adhesion for surface treated rice husk flour-filled polypropylene bio-composites," Mokchae Konghak, vol. 34, no. 3, pp. 38-45, 2006. 
[6] T. S. Lee, S. G. Lee, and K. H. Song, "Natural fiber reinforced biocomposites and biodegradation," Fashion Information and Technology, vol. 7, pp. 10-21, 2010.

[7] S. Singh, D. Y. Sasaki, J. Cesarano III, and A. J. Hurd, "Nanometer pores in ultrathin silica films prepared by self-assembly of organic spacers in an alkylsiloxane monolayer," Thin Solid Films, vol. 339, no. 1-2, pp. 209-215, 1999.

[8] J. Lai, B. Sunderland, J. Xue et al., "Study on hydrophilicity of polymer surfaces improved by plasma treatment," Applied Surface Science, vol. 252, no. 10, pp. 3375-3379, 2006.

[9] V. Kotal, V. Svorc1k, P. Slepicka et al., "Gold coating of poly(ethyleneterephthalate) modified by argon plasma," Plasma Process and Polymers, vol. 4, no. 1, pp. 69-76, 2007.

[10] S.-H. Seo, S. Chang, Y.-E. Yoo, and J. D. Chung, "Surface characteristics of polymer material treated by atmospheric pressure plasma," Korean Journal of Air-Conditioning and Refrigeration Engineering, vol. 22, no. 5, pp. 282-288, 2010. 

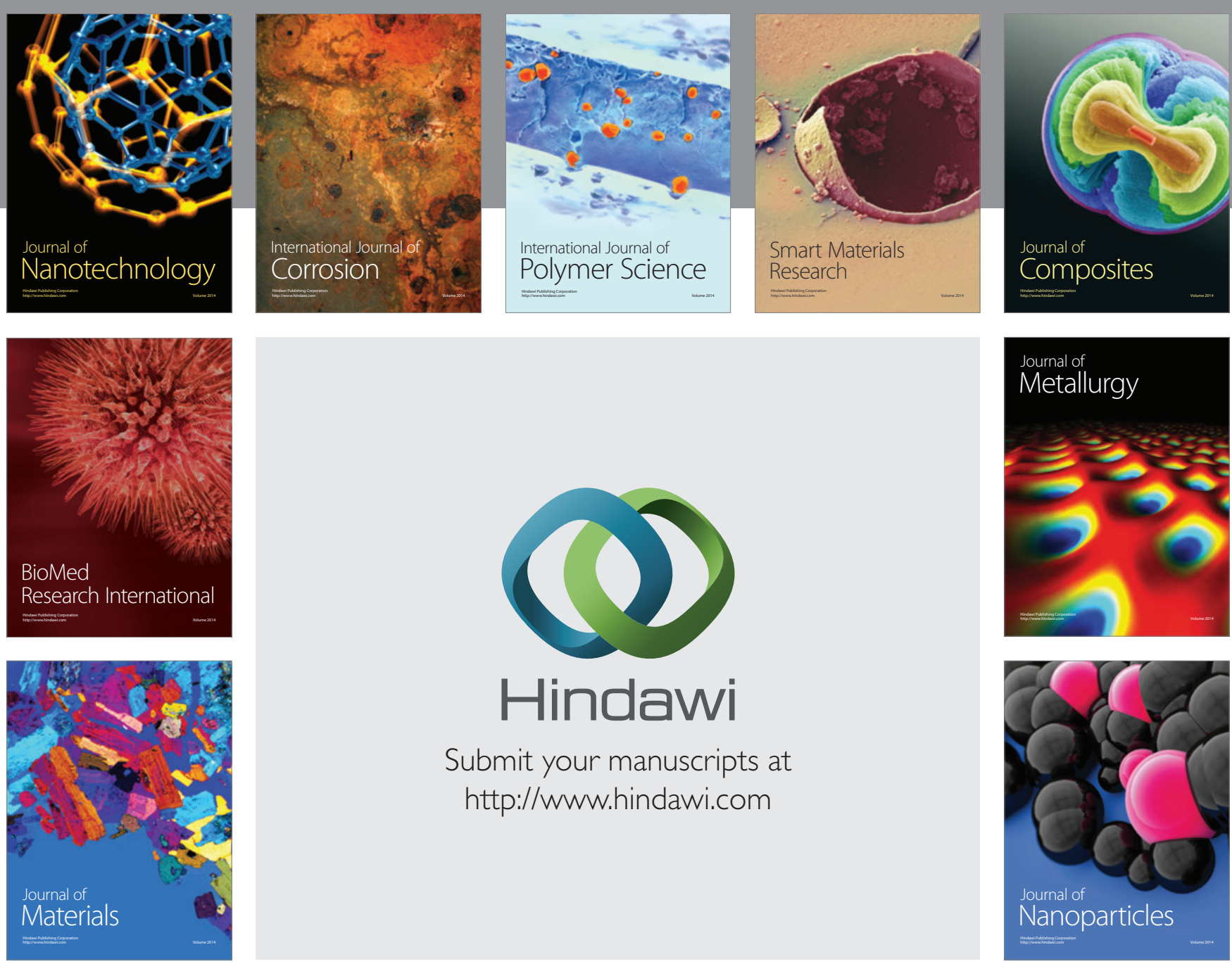

Submit your manuscripts at http://www.hindawi.com
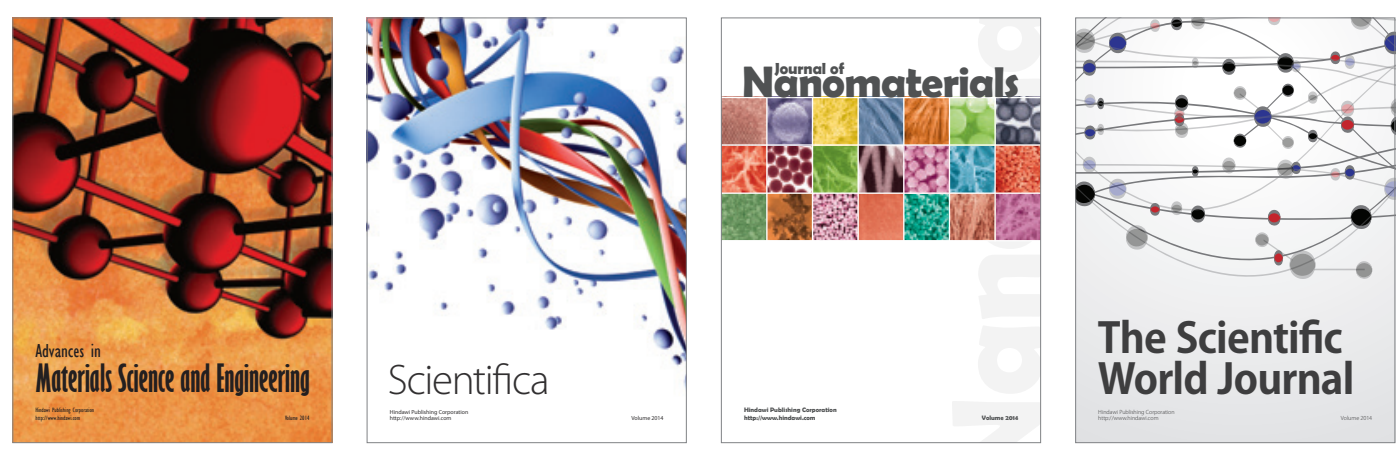

\section{The Scientific World Journal}
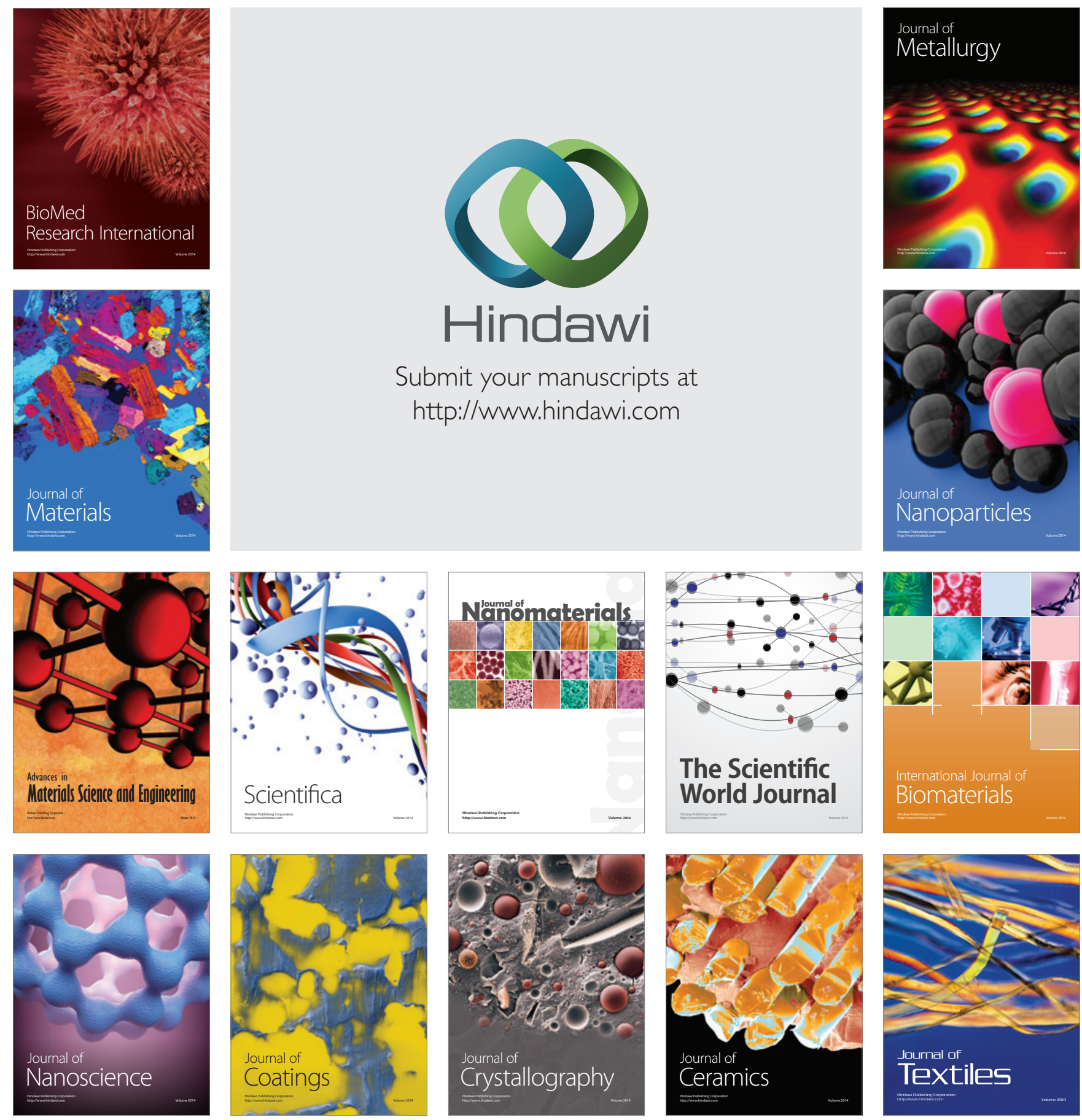